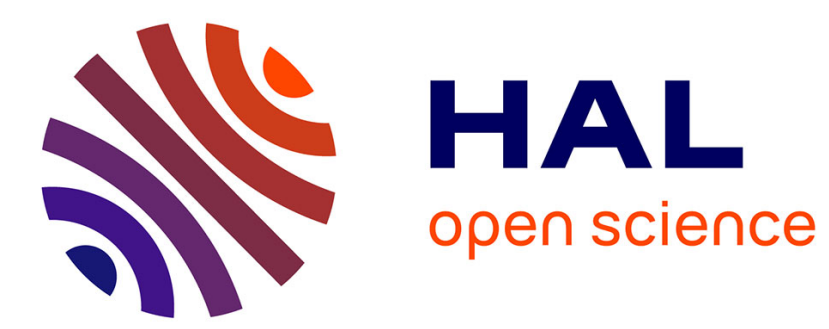

\title{
Local Arrangements in Fe-31\% at. Pd above Ms
}

J. Felten, J. Cohen

\section{To cite this version:}

J. Felten, J. Cohen. Local Arrangements in Fe-31\% at. Pd above Ms. Journal de Physique IV Proceedings, 1995, 05 (C8), pp.C8-485-C8-490. 10.1051/jp4:1995874 . jpa-00254123

\section{HAL Id: jpa-00254123 https://hal.science/jpa-00254123}

Submitted on 1 Jan 1995

HAL is a multi-disciplinary open access archive for the deposit and dissemination of scientific research documents, whether they are published or not. The documents may come from teaching and research institutions in France or abroad, or from public or private research centers.
L'archive ouverte pluridisciplinaire HAL, est destinée au dépôt et à la diffusion de documents scientifiques de niveau recherche, publiés ou non, émanant des établissements d'enseignement et de recherche français ou étrangers, des laboratoires publics ou privés. 


\title{
Local Arrangements in $\mathrm{Fe}-31 \%$ at. Pd above $\mathrm{M}_{\mathbf{S}}$
}

\author{
J.J. Felten and J.B. Cohen*
}

Containerless Research Inc., 910 University Place, Evanston, IL 60201, U.S.A.

* Department of Materials Science \& Engineering, Robert R. McCormick School of Engineering and Applied Science, Northwestern University, 2225 North Campus Drive, Evanston, IL 60208, U.S.A.

\begin{abstract}
The diffuse $\mathrm{x}$-ray scattering from an $\mathrm{Fe}-31$ at. pct. Pd single crystal quenched from $1173 \mathrm{~K}$ was analyzed to obtain information about the local atomic environment around each atom including the local chemical order and the average atomic displacements from the normal lattice positions. To better reveal the meaning of these average quantities, computer simulations were employed to provide the structure using these values. The results indicate an interconnected distribution of ordered and clustered regions, no more than a few atoms in dimension, and only slightly more developed than for a random arrangement. The pattern of atomic displacements from the average lattice is revealed to be small embryos of a displacement array similar to the FCT martensitic phase and aligned in $\langle 110\rangle$ directions, which may be the premartensitic phase that has been predicted by several authors.
\end{abstract}

\section{INTRODUCTION}

Displacive transformations play a key role in determining the properties of certain materials, most noticeably steels. In this study we focus on this transformation in the Fe-Pd system, which occurs upon quenching to avoid the miscibility gap. The face-centered cubic structure transforms into a facecentered tetragonal (FCT)one [1]. This transformation is weakly first order, and therefore a metastable product might occur just above the $\mathrm{M}_{\mathbf{S}}$. In fact, premonitory effects have been reported in this vicinity, one of which is the so-called tweed microstructure observed in the transmission electron microscope [2]. Detailed investigations with the electron microscope have been performed by Oshima et. al. [2-4] and Muto et. al. [5-9]. Also accompanying the tweed are streaks of diffuse scattering. Both effects are also seen in precipitation reactions [10], and in both cases are attributed to a localized build up of strain, either due to a coherent phase, a transformation product, or a static strain wave. Oshima et. al. [2-4] observed that tweed occurred in the Fe-Pd system in alloys of less than 32 atomic pct. Pd, where the martensite phase forms, and in equiatomic Fe-Pd, for which there is an order-disorder transformation (with an appreciable volume change), from FCC to FCT. These authors came to the conclusion that this tweed was caused by small domains of the tetragonal phase which produced static displacements on $\{110\}$ planes in $<110\rangle$ directions in the parent phase. Muto et. al. [9] performed computer simulations to try to produce tweed images and found that a model based on small (tetragonal) domains reproduced the tweed microstructure.

Employing ultrasonic techniques and inelastic neutron scattering Muto et. al. [5] found softening of the elastic constant $C^{\prime}$ with little change in $\mathrm{C}_{44}$ as $\mathrm{M}_{\mathbf{S}}$ was approached. Thus, as the temperature is decreased, the lattice is increasingly easy to shear along $\langle 110\rangle$ directions. In addition, Matsui and Adachi [11] found that the direction of magnetostriction changed from $\langle 111\rangle$ to $\langle 100\rangle$ at the $\mathbf{M}_{\mathbf{S}}$. Oshima[2] thought this suggested that the distortion was driven by magnetic forces.

Seto et. al. [12,13] observed an apparent intermediate phase via X-ray diffraction just slightly below $\mathrm{M}_{\mathrm{S}}$, and suggested that it was another FCT phase, coherent with the final phase. Khatchaturyan et. al. [14] later described this as "adaptive martensite", a finely twinned region with the twin variants 
periodically arrayed to create a pseudo-orthorhombic structure. In applying this concept to the Ni-Al and $\mathrm{Fe}-\mathrm{Pd}$ systems, they found good agreement with the predicted lattice parameters.

Hong and Olson [15] showed theoretically that $M_{S}$ was dependent on the local density of electronic states in the Fe-Pd system, and, as a result, followed the Pd concentration. The softening of $\mathrm{C}^{\prime}$ was found to be due to magnetoelastic coupling. Their model led to a calculated diffusionless phase diagram in good agreement with the actual one. In addition, Kartha [16] considered the role of compositional inhomogeneities. The transformation temperature is sharply dependent on composition, so that regions with different chemical compositions might be expected to transform at different temperatures, and he pursued this point, showing that such regions might be responsible for the lattice softening and the tweed microstructure. It might reasonably be asked if there really are composition fluctuations tied to the lattice distortions, and this is one of the subjects of this investigation.

\section{EXPERIMENTAL DETAILS}

A coarse grained Fe-Pd ingot of approximately 30 at. pct. $\mathrm{Pd}$ was grown by the Ames Laboratory. A large grain was cut form one part of the ingot and the composition determined to be 31(0.1) at. pct. Pd by EDAX in an SEM. (Another piece of the ingot that had been analyzed chemically was used as a standard.) After homogenization at $1423 \mathrm{~K}$ for five days in a sealed quartz tube evacuated to $10^{-6}$ Torr, a final solution treatment at $1173 \mathrm{~K}$ for six hours was employed, and the ampule was then quenched in oil. Oxidation and cold-worked material near the surface were removed by electropolishing.

Chemical order and the first and second order moments of the atomic displacements from the average lattice sites each lead to intensity contributions that differ in symmetry in reciprocal space. By making these measurements in a volume in reciprocal space at many symmetry-related points, these intensity components can be separated, following the well known Georgopoulos-Cohen procedure [17], and then Fourier inverted to yield:

1)the Warren short-range order parameters,

$$
\alpha_{l m n}=1-P_{l m n}^{F e-P d} / C_{P d}
$$

where $P_{l m n}^{F e-P d}$ is the conditional probability of finding an Pd atom at the end of the interatomic vector $\ell m n$ if there is an $\mathrm{Fe}$ at the beginning of this vector, and $C_{P d}$ is the atomic fraction,

2) the moments $\left\langle X_{l m n}^{F e-P d}\right\rangle,\left\langle Y_{l m n}^{F e-P d}\right\rangle,\left\langle Z_{l m n}^{F e-P d}\right\rangle$ which are the orthogonal components of displacements from the mean lattice, averaged over all $\ell m n$ vectors and similarly for Fe-Fe and Pd-Pd pairs, and as well the second moments, $\left\langle\left(X_{l m n}^{F e-P d}\right)^{2}\right\rangle,\left\langle\left(X_{0}^{F e} Y_{l m n}^{P d}\right)\right\rangle$ etc. Because these last moments include first order thermal vibrations as well as static displacements, we report only the mean terms, as they involve only the static displacements; the thermal contributions average to zero.

The experimental arrangement included a sealed Co x-ray tube, and a singly bent graphite monochromator bent to focus vertically at the receiving slits to minimize the sampling volume. The crystal was mounted inside an evacuated Be hemispherical chamber to minimize air scattering and this was mounted on a quarter-circle goniometer on a G.E. diffractometer.

\section{RESULTS AND DISCUSSION}

\subsection{Local chemical order}

The first point to note in table $I$ is the closeness of the $\alpha_{000}$ term to unity, its defined value. The probability in Eq. 1 must be zero at $\ell m n$ all equal to zero, because no two atoms can occupy the same space at the same time. This value is a good test of the analysis procedures employed for putting the data on an absolute scale and the various corrections.

The first and second neighbor SRO parameters in table I imply local ordering, that is, the first nearest neighbors tend to be of different species while the second nearest neighbors tend to be of like 
species. Furthermore, after about the fourth nearest neighbor shell the magnitude of the alphas is essentially zero, suggesting that the atomic arrangement is effectively random beyond this distance. In order to understand the nature of this ordering more clearly, we employed computer simulation, first developed by Gehlen and Cohen [18], to create a structure in $32 \times 32 \times 32$ FCC unit cells (131072 atoms) which match the measured short-range order parameters, as shown in Table I.

Ordered and clustered atoms in the simulated lattice were then defined taking into account that atoms near the edges of any clusters or small ordered regions could not be in a perfect ordered or clustered environment. Fe atoms were marked as ordered if there were four Pd atoms in the first shell and zero or one in the second, whereas for Pd these numbers were two or fewer Pd in the first shell and three or more $\mathrm{Pd}$ in the second shell. The appearances of both the actual alloy and a random configuration were quite similar, small ordered irregular volumes, often attached to small antiphase regions or clusters of $\mathrm{Fe}$ (see Fig. 1a). However, $18 \mathrm{pct}$. of the $\mathrm{Pd}$ atoms were ordered in the actual alloy and 10 pct. of the $\mathrm{Fe}$, compared to 8 pct. of each in the random configuration. The growth of the number of these regions (not their size) may have occurred on crossing the miscibility gap, as only a fraction of a second is needed for diffusion over one or two unit cells as calculated from available data on diffusion in $\mathrm{Fe}$ and $\mathrm{Pd}[19]$.

To gain a better understanding for the local composition fluctuations, a $5 \times 5$ unit cell column perpendicular to the (100) planes was chosen at random in the simulations, and the composition on each plane (50 atoms) was calculated through the column, as well as the fraction of ordered $\mathrm{Fe}$ and $\mathrm{Pd}$ atoms. Within such a small column the composition can vary from 20 to 60 pct. Pd in the actual alloy (less in the random case), and since a change in composition of only 3 pct. changes the $M_{S}$ by 200 degrees, this fluctuation can be quite significant.

\subsection{Atomic displacements}

The average atomic displacements are given in Table $I$ for various interatomic vectors and for the three kinds of pairs. An EXAFS study is available for Fe-30 at. pct. Pt for comparison [20], but this technique can only reliably sample the first neighbor shell. As can be seen in this table, the displacements are significant only for this shell in Fe-Pd. Furthermore, the magnitude of the displacements is about 2 pet for Pd-Pd and Fe-Pd pairs, similar to that for Fe-Pt and Pt-Pt pairs in the Fe-Pt study. Note that the Fe-Fe nearest neighbor distance is displaced transverse to the $\langle 110\rangle$ direction, and is nearly unchanged in length, the Pd-Pd distance is shortened along this vector, and the Fe-Pd distance is lengthened along the $<100>$ direction. (Such details are not available from EXAFS, which gives only the change in magnitude of the spacing.)

The displacements were also simulated, employing a new program developed by Kyobu, Murata and Morinaga [21]. The program first carries out a simulation of the local order and then simulates the displacements to yield the averages in Table I; the first twelve SRO and displacement terms were employed. (The simulation of local order was compared to the one described above and found to yield similar results.) To make the displacements easier to study those above one pct. were magnified by a factor of ten in the images produced (see Fig 1b).

Most of the displacements are in $\langle 100\rangle$ directions, despite the fact that the only observed value of significance is the average value in $\langle 110\rangle$ directions. A change from the FCC to the FCT phase in this system can in fact be accomplished by an expansion along two $<100>$ directions and a contraction along the third $\langle 100\rangle$ direction. We might be sampling the embryonic stage of this transformation.

The simulations were searched to discern whether or not the distorted regions were forming in Pd or Fe rich regions. We defined a displaced atom as one with a displacement magnitude of 2.5 pct. of the lattice parameter or greater. The results showed the displacements had a tendency to be associated with $\mathrm{Fe}$ atoms rather than with the $\mathrm{Pd}$. In addition, a higher percentage of the clustered $\mathrm{Fe}$ atoms were displaced over the ordered Fe or Pd atoms. Thus, the displacements do tend to occur in Pd deficient regions. 


\section{CONCLUSIONS}

1)The measured short-range order parameters for Fe-31 at. pct. Pd quenched from $1173 \mathrm{~K}$ indicate that short-range order exists, rather than clustering. The ordering is not very large and the ordered regions are only a few unit cells in size. While these regions are three dimensional, they do not have any simple shape.

2)The composition fluctuates between 20-60 at. pct. Pd in small 50 atom regions in the alloy.

3)Regions of higher Pd composition tend to have a higher degree of order.

4)There are small distorted regions which may be the FCT martensite nucleii.

5)The displacements of individual atoms tend to be in $<100>$ type directions.

6)The displacements tend to be larger in the $\mathrm{Pd}$ deficient regions.

7)The displaced regions are in bands along $\langle 110\rangle$ directions.

\section{ACKNOWLEDGMENTS}

This work was sponsored by NSF under grant number DMR8417961. We thank Dr. Bruce MacDonald for his continued interest and advice. We would also like to express our gratitude to Dr. Po Hong and Prof. G.B. Olson of Northwestern University for providing the sample and for the excellent discussions they contributed to this work. The authors also thank J. Kyobu, Y. Murata and M. Morinaga of Toyohashi University at the time (M. Morinaga now at Nagoya University) for providing us with their new program on displacements, and Dr. M. Kimura, Nippon Steel Co. for translating the instructions.

\section{REFERENCES}

[1] Kubaschewski, O., Iron-Binary Phase Diagrams $1^{\text {st }}$ ed. (Springer-Verlag, Berlin 1982) pp. 88-91.

[2] Oshima R., Muto S., Fujita F.E., Trans. JIM, 333 (1992), 197-202.

[3] Oshima R., Tanaka K., Taniyama A., Fujita F.E., Materials Science Forum, 56-58 (1990), 175-180.

[4] Oshima R., Sugiyama M., Fugita F.E., Metall. Trans A, 19A (1988), 803

[5] Muto S., Oshima R., Fujita F.E., Acta Metall., 38, 4 (1990), 685-694.

[6] Muto S., Oshima R., Fujita F.E., Materials Science Forum, 56-58 (1990), 65-70.

[7] Muto S., Takeda S., Oshima R., Fujita F.E., Jpn. J. Appl. Phys., 27 (1988), L1387-L1389.

[8] Muto S., Takeda S. Oshima R. Fujita F.E., Materials Science Forum, 56-58 (1990), 45-50.

[9] Muto S., Takeda S. Oshima R., Fujita F.E., J. Phys.: Condens Matter, 1 (1989), 9971-9983.

[10] Robertson, J.M., Wayman, C.M., Phil. Mag. A, 48 (1983), 443-67.

[11] Matsui M., Adachi K., Physica B, 161 (1989), 53-59.

[12] Seto H., Noda Y., Yamada Y., Materials Science Forum, 56-58 (1990), 77-82.

[13] Seto H., Noda Y., Yamada Y., J. Phys. Soc. Jpn., 593 (1990), 965-977.

[14] Khatchaturyan A.G. Shapiro S.M. Semenovskaya S., Phys. Rev. B, 43 (1991), 10832.

[15] Hong P., Olson G.B., J. Magn. Magn. Mater., 129 (1994), 191-199.

[16] Kartha S., Ph.D. Thesis, Princeton University, Princeton University (1993).

[17] Schwartz L.H., Cohen J.B., Diffraction from Materials (Springer-Verlag, Heidelberg 1987) pp. 402-422.

[18] Gehlen, P.C., Cohen, J.B., Phys. Rev., 139A (1965), 844-855.

[19] Porter D.A., Easterling K.E., Phase Transformations in Metals and Alloys (Chaptman and Hall, New York 1990) pp. 78-79.

[20] Maruyama H., Shirai K,. Maeda H., Liu W., Yamada O., J. Phys. Soc. Jpn., 56 (1987), 4377.

[21] Kyobu J., Murata Y., Morinaga M., J. Appl. Crystall., 27 (1994) 772-781. 


\begin{tabular}{|c|c|c|c|c|c|}
\hline $2(m n)$ & SRO Parameter & $<X(F e-P d)>$ & $<Y(F e-P d)>$ & $<Z(F e-P d)\rangle$ & $\begin{array}{c}\text { Simulated } \\
a\end{array}$ \\
\hline 000 & $1.0276(0.0372)$ & $0.0000(0.0000)$ & $0.0000(0.0000)$ & $0.0000(0.0000)$ & \\
\hline 110 & $-0.0760(0.0151)$ & $0.0009(0.0038)$ & $0.0000(0.0000)$ & $0.0000(0.0000)$ & -0.0760 \\
\hline 200 & $0.0951(0.0110)$ & $0.0009(0.0038)$ & $0.0000(0.0000)$ & $0.0000(0.0000)$ & 0.0953 \\
\hline 211 & $-0.0006(0.0064)$ & $0.0008(0.0018)$ & $-0.0005(0.0008)$ & $-0.0005(0.0008)$ & -0.0088 \\
\hline 220 & $0.0547(0.0079)$ & $0.0020(0.0012)$ & $0.0020(0.0012)$ & $0.0000(0.0000)$ & 0.0547 \\
\hline 310 & $0.0097(0.0090)$ & $0.0024(0.0016)$ & $-0.0010(0.0012)$ & $0.0000(0.0000)$ & 0.0097 \\
\hline 222 & $0.0008(0.0095)$ & $-0.0004(0.0010)$ & $-0.0004(0.0010)$ & $-0.0004(0.0010)$ & 0.0359 \\
\hline 321 & $0.0022(0.0046)$ & $0.0001(0.0009)$ & $0.0021(0.0011)$ & $-0.0017(0.0010)$ & -0.0077 \\
\hline 400 & $-0.0269(0.0124)$ & $-0.0046(0.0018)$ & $0.0000(0.0000)$ & $0.0000(0.0000)$ & -0.0268 \\
\hline 330 & $-0.0011(0.0080)$ & $-0.0006(0.0011)$ & $-0.0006(0.0011)$ & $0.0000(0.0000)$ & -0.0012 \\
\hline 411 & $0.0103(0.0069)$ & $-0.0013(0.0010)$ & $-0.0004(0.0007)$ & $-0.0004(0.0007)$ & 0.0103 \\
\hline 420 & $0.0155(0.0059)$ & $-0.0018(0.0011)$ & $0.0010(0.0012)$ & $0.0000(0.0000)$ & 0.0155 \\
\hline 332 & $-0.0039(0.0090)$ & $0.0007(0.0010)$ & $0.0007(0.0010)$ & $-0.0009(0.0011)$ & 0.0003 \\
\hline 422 & $-0.0058(0.0057)$ & $-0.0001(0.0011)$ & $-0.0010(0.0008)$ & $-0.0010(0.0008)$ & \\
\hline 431 & $0.0018(0.0037)$ & $-0.0007(0.0008)$ & $0.0004(0.0009)$ & $-0.0009(0.0007)$ & \\
\hline 510 & $-0.0021(0.0079)$ & $0.0000(0.0009)$ & $-0.0009(0.0012)$ & $0.0000(0.0000)$ & \\
\hline
\end{tabular}

Table I. Parameters obtained by diffuse scattering analysis of $\mathrm{Fe}-\mathrm{Pd}$ pairs. (means used as input into the simulation program) 


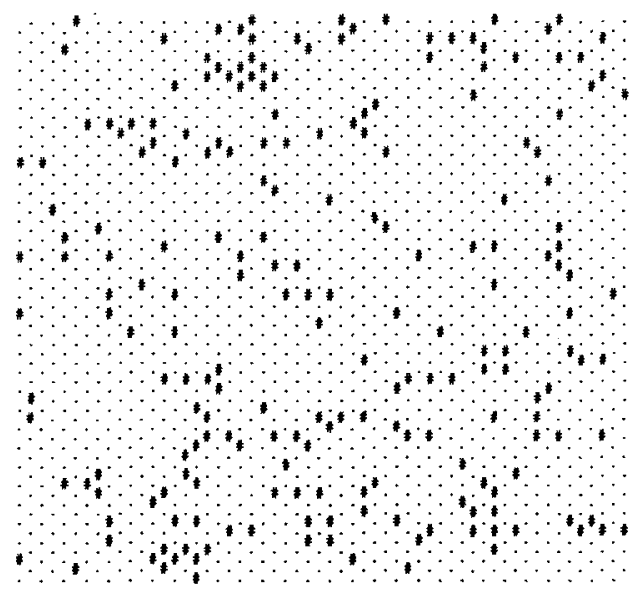

(a)

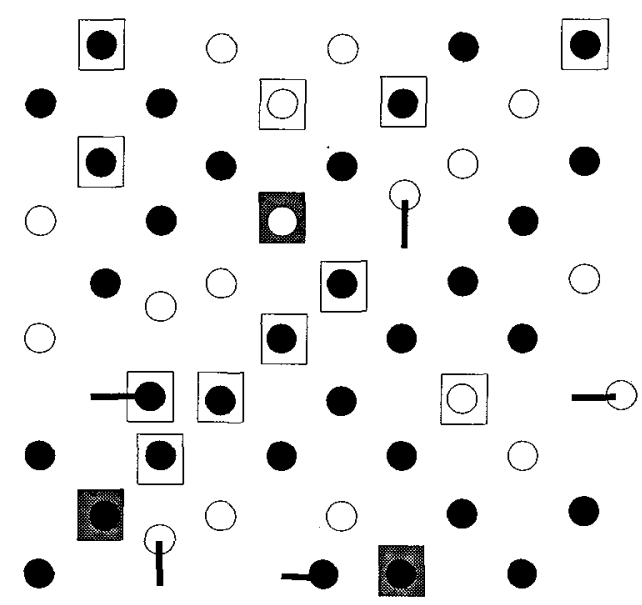

(b)

Figure 1. (a) Simulated structure "." = disordered atom, "\#" = ordered atom. (b) Sample output from the simulation of displacements with the displacements magnified. Displacements in the plane of the figure are given by lines, with the beginning position at one end and the final atom position at the other. Displacements greater than 1.5 percent of the lattice parameter and perpendicular to the plane are indicated by boxes. A filled box indicates an upward displacement while a clear box indicates a downward displacement. An open circle represents a $\mathrm{Pd}$ atom while a closed circle represents an $\mathrm{Fe}$ atom. 